\title{
COMPLEMENT SYNTHESIS IN THE DISEASED KIDNEY AND ITS ROLE IN RENAL DAMAGE
}

\author{
KHATUN $M^{1}$, NURUNNABI ASM ${ }^{2}$, ARA $\mathrm{S}^{3}$, RAHMAN M ${ }^{4}$
}

\begin{abstract}
:
Renal biopsy tissues were taken from 142 suspected glomerulonephritic patients who were admitted into the Department of Nephrology of Bangabandhu Sheikh Mujib Medical University (BSMMU), Dhaka and Combined Military Hospital (CMH), Dhaka Cantonment, Dhaka. The tissues were processed for both Light Microscopy (LM) and Direct Immunofluorescence (DIF) studies.

The study was done in the Department of Anatomy, Bangabandhu Sheikh Mujib Medical University (BSMMU), Dhaka and Armed Forces Institute of Pathology (AFIP), Dhaka Cantonment, Dhaka, from March to December 1999.

Seven histopathological types of glomerulonephritis were identified with LM and another one type i.e. IgA Nephropathy was identified exclusively by using DIF. Diffuse immunofluorescence positivity was found in $44.36 \%$ cases. C3 components were found in all cases irrespective of the histopathological type of glomerulonephritis. Immune complex deposits were observed in immunofluorescence both in the mesangium and the glomerular basement membrane (GBM) with more generalized and less scattered distributions. Immunoglobulins (Ig) were tested for IgG, IgA and IgM. IgG was found the most common (74.60\%) among immune complex deposits. Notable LM features include proliferation of mesangial cells, expansion of mesangial matrix, thickening of GBM, infiltration of glomerular macrophages, platelets and neutrophil and crescent formation. The presence of IgG in the mesangium of the kidney of the glomerulonephritic patient suggests a role of $\operatorname{Ig} G$ in the inflammatory process. There is also evidence that $C 3$ is synthesized within the glomeruli of the patients with glomerulonephritis. Finding the role of the complement components in pathogenesis of glomerulonephritis, a keen observation is needed to determine the extent of local complement synthesis and their involvement in tissue injury process.
\end{abstract}

Key words: Complement synthesis, immune complex, glomerulonephritis, renal biopsy.

J Dhaka Med Coll. 2011; 20(1) : 15-19.

\section{Introduction:}

The renewed appreciation of the role of the complement system as a mediator and marker of renal damage has led to numerous novel investigations in the field of complement and renal disease ${ }^{1}$. The complement system is a complex set of soluble and membrane-bound proteins and constitutes a major element of the innate immune system ${ }^{2}$. Complement may play both a beneficial as well as a harmful role in renal disease. Complement deposition is detected in kidney biopsies obtained from patients with various forms of renal disease.
Except for type-II membranoproliferative glomerulonephritis, complement deposition is usually accompanied by the deposition of immunoglobulins ${ }^{1}$.

Direct immunofluorescence (DIF) microscopic study of renal biopsy material has proved to be a valuable supplement to clinical examination and conventional histomorphological studies ${ }^{3}$. Immunofluorescence microscopy is particularly helpful in determining the patterns of deposition in renal disease ${ }^{4}$. Firstly, it helps to identify granular deposits of immunoglobulins, a hallmark of immunocomplex nephritis ${ }^{4,5,6}$.

1. Dr. Monira Khatun, Associate Professor, Department of Anatomy, Dhaka Medical College, Dhaka.

2. Dr. Abu Sadat Mohammad Nurunnabi, Lecturer, Department of Anatomy, Dhaka Medical College, Dhaka.

3. Prof. Shamim Ara, Professor and Head, Department of Anatomy, Dhaka Medical College, Dhaka.

4. Dr. Mustafizur Rahman, Associate Professor, Department of Anaesthesiology, National Institute of Cardiovascular Diseases (NICVD), Dhaka.

Correspondence : Dr. Monira Khatun, Associate Professor, Department of Anatomy, Dhaka Medical College, Dhaka. Cell Phone: +8801715408900, Email: khatunm59@yahoo.com 
Secondly, it helps to indentify granular deposits along the glomerular basement membrane ${ }^{5,6}$. Finally, it also determines the paucity or absence of immunoglobulins ${ }^{5}$. Virtually, any antigen can be detected in mixed tissue sections or in live suspensions by immunofluorescence technique. However, it is the combination of great sensitivity and specificity together with the use of histologic technique that makes immunofluorescence technique so useful in clinical practice ${ }^{7}$. Therefore, the present study was an attempt to correlate the findings of immunofluorescence technique and light microscopic (LM) study of renal biopsy in diagnosis of different glomerular disease.

\section{Materials and Methods:}

Renal biopsy tissues were taken from 142 suspected glomerulonephritic patients who were admitted into the Department of Nephrology of Bangabandhu Sheikh Mujib Medical University (BSMMU), Dhaka, and Combined Military Hospital (CMH), Dhaka Cantonment, Dhaka. The tissues were processed for both Light Microscopy (LM) and Direct Immunofluorescence (DIF) studies.

The study was done in the Department of Anatomy, Bangabandhu Sheikh Mujib Medical University (BSMMU), Dhaka, and Armed Forces Institute of Pathology (AFIP), Dhaka Cantonment, Dhaka, from March to December 1999.

Renal biopsy: After taking a written informed consent and ensuring all aseptic precautions, percutaneous renal biopsy was done in each patient in the prone position by using a disposable 'tru-cut' biopsy needle, under local anaesthesia.

Tissue preparation for light microscopy: The light microscopic studies were done on paraffinembedded sections, which were fixed in 10\% purified buffered formalin. Sections were cut at $2-5 \mu \mathrm{m}$. Both Haematoxylin \& Eosin (H\&E) and Periodic Acid-Schiff (PAS) stains were used for each biopsy material. The compound light microscope used for the study was Olympus CHB (made in Japan).
Tissue preparation for immunofluorescence microscopy: The reagent used was fluorescein iso-thiocyanate (FITC) conjugated antihuman monoclonal antibodies for IgG, IgA and C3 component of the complement complex. The tissues were sectioned on cryostat machine at $2-3 \mu \mathrm{m}$, stained with FITC, and viewed through barrier filters (OG-4), along with ultraviolet activating filter (UG-1) and heat absorption filter (KG-1), with an ultraviolet light source (HB-200). The fluorescent microscope used for the study was NIKON Labophot-2 (model-661012, made in Japan). The intensity of fluorescence was graded arbitrarily as none $(-)$, trace $(+/-), 1(+), 2(++)$ and $3(+++)^{3}$.

Photomicrographs of the renal sections: The photomicrographs were taken by using Yashica camera (made in Japan) and printed on Kodak colour photopaper (made in USA).

Data analysis: All the relevant information about the patients and their histopathological reports were checked and noted down on the predesigned data sheet for further analyses with MS-Excel.

\section{Results:}

Seven histopathological types of glomerulonephritis were identified with LM and another one type i.e. IgA Nephropathy was identified exclusively by using DIF. Diffuse immunofluorescence positivity was found in $44.36 \%$ cases. C3 components were found in all cases irrespective of the histopathological type of glomerulonephritis. Immune complex deposits were observed in immunofluorescence both in the mesangium and the glomerular basement membrane (GBM) with more generalized and less scattered distributions. Immunoglobulins (Ig) were tested for IgG, IgA and IgM. IgG was found the most common $(74.60 \%)$ among immune complex deposits. Notable LM features include proliferation of mesangial cells, expansion of mesangial matrix, thickening of GBM, infiltration of glomerular macrophages, platelets and neutrophil and crescent formation. The presence of IgG in the mesangium of the kidney of the glomerulonephritic patient suggests a role of IgG in the inflammatory process. There is also evidence that $\mathrm{C} 3$ is synthesized within the glomeruli of the patients with glomerulonephritis. The results are shown in table-I, II \& III. 
Table-I

Frequency of cases with positive features in light microscopy (LM) and direct immunofluorescence (DIF) microscopy in different histomorphological types of glomerulonephritis

\begin{tabular}{lccccc}
\hline Histomorphological & \multicolumn{2}{c}{ Cases diagnosed through LM } & & \multicolumn{2}{c}{ DIF positive cases } \\
\cline { 2 - 3 } \cline { 5 - 6 } type of GN & Number & \% of total cases & & Number & \% of total cases \\
\hline MsPGN & 39 & 27.46 & 16 & 41.02 \\
RPGN & 2 & 1.40 & 2 & 100 \\
MGN & 10 & 7.04 & 9 & 90 \\
MCD & 19 & 13.38 & - & - \\
FSGS & 16 & 11.26 & 8 & 50 \\
MPGN & 8 & 5.65 & 7 & 87.5 \\
FPGN & 37 & 26.05 & 10 & 100 \\
IgA Nephropathy & - & - & 11 & \\
Total & 142 & & 63 & \\
\hline
\end{tabular}

Table-II

Frequency of immunofluorescence deposits for different sites, patterns and distribution in different histomorphological types of glomerulonephritis (GN)

\begin{tabular}{|c|c|c|c|c|c|c|c|c|}
\hline \multirow{3}{*}{$\begin{array}{l}\text { Histomorphological } \\
\text { type of GN }\end{array}$} & \multirow{3}{*}{$\begin{array}{l}\text { No. of } \\
\text { DIF +ve } \\
\text { cases }\end{array}$} & \multirow[b]{3}{*}{ Ms } & \multicolumn{4}{|c|}{ Frequency of deposit } & \multicolumn{2}{|c|}{ Distribution } \\
\hline & & & \multicolumn{2}{|c|}{ Site of deposit } & \multicolumn{2}{|c|}{ Pattern of deposit } & \multirow[b]{2}{*}{ Scattered } & \multirow[b]{2}{*}{ Generalized } \\
\hline & & & GBM & $\begin{array}{c}\text { Both Ms } \\
\text { only }\end{array}$ & $\begin{array}{c}\text { Granular } \\
\text { only }\end{array}$ & $\begin{array}{l}\text { Linear } \\
\& \text { GBM }\end{array}$ & & \\
\hline MsPGN & 16 & 16 & - & - & 16 & - & 4 & 12 \\
\hline RPGN & 2 & 2 & - & - & 2 & - & - & 2 \\
\hline $\mathrm{MGN}$ & 9 & - & 9 & - & 9 & - & 3 & 6 \\
\hline MCD & - & - & - & - & - & - & - & - \\
\hline FSGS & 8 & 8 & - & - & 8 & - & 5 & 3 \\
\hline MPGN & 7 & - & - & 7 & 7 & - & - & 7 \\
\hline FPGN & 10 & 10 & - & - & 10 & - & 2 & 8 \\
\hline IgA Nephropathy & 11 & 11 & - & - & 11 & - & 2 & 9 \\
\hline Total & 63 & 47 & 9 & 7 & 63 & - & 16 & 47 \\
\hline
\end{tabular}

DIF: Direct immunofluorescence

Ms: Mesangium

GBM: Glomerular basement membrane

Table-III

Frequency of different intensities of immunofluorescence determining the immune complex deposition.

\begin{tabular}{lcccc}
\hline Type of deposit & No. of DIF & \multicolumn{3}{c}{ Frequency of intensity } \\
\cline { 3 - 5 }+ ve cases & High & Moderate & Mild \\
\hline IgG + C3 & 47 & $5(10.63 \%)$ & $29(61.70 \%)$ & $13(18.18 \%)$ \\
IgA + C3 & 11 & $2(18.18 \%)$ & $7(63.64 \%)$ & $2(18.18 \%)$ \\
IgM + C3 & 5 & - & $5(100 \%)$ & - \\
\hline
\end{tabular}




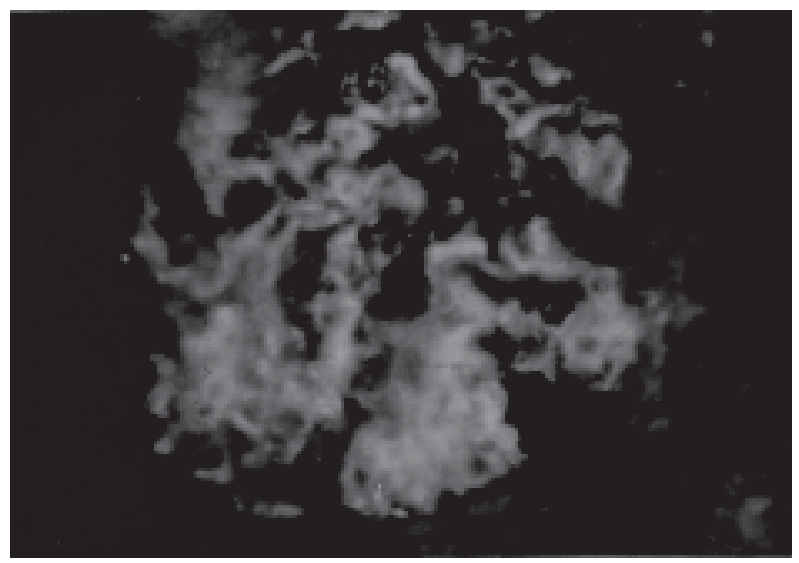

Fig.-1: Photomicrograph showing membranous glomerulonephritis with granular deposition of IgM in the mesangium (DIF stain $x$ 1600).

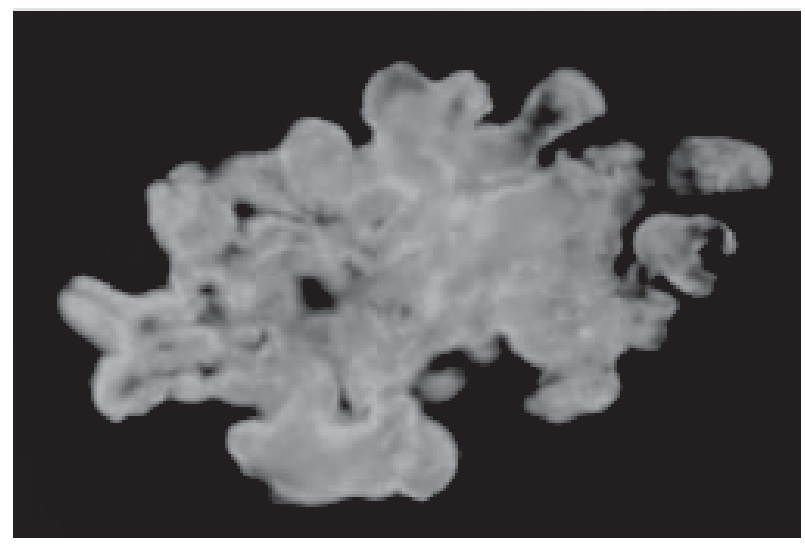

Fig.-2. Photomicrograph showing membranous glomerulonephritis with granular deposition of IgG along the glomerular basement membrane (DIF $\operatorname{stain} \times 1600)$.

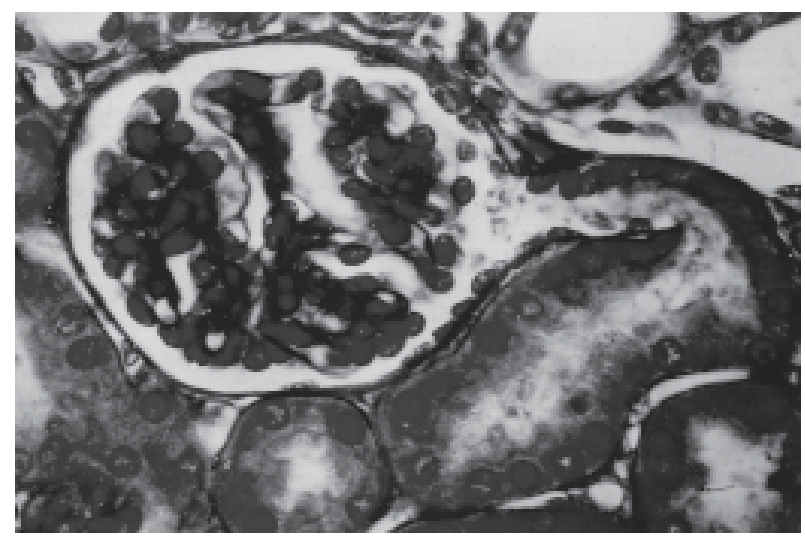

Fig.-3: Photomicrograph showing multilayered crescent formation in the glomerulus. The hypercellular glomerulus is compressed by the presence of cellular crescent in Bowman's space (H\& E stain $x$ 800).

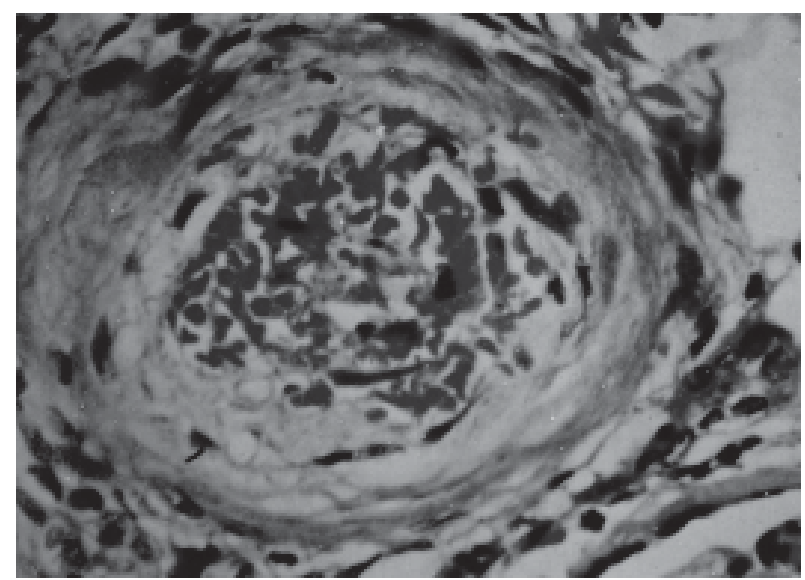

Fig.-4: Photomicrograph showing only minimal increase in mesangial cells and mild increase in mesangial matrix and thickness of capillary basement membrane (PAS stain $x$ 800).

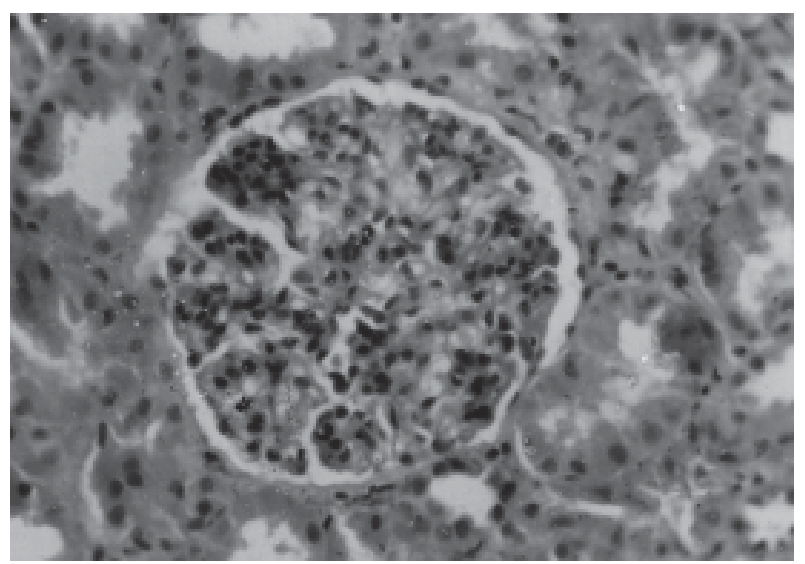

Fig.-5: Photomicrograph showing involvement of an enlarged glomerulus both focally and segmentally, with hypercellularity of both matrix and cells ( $H \&$ \& stain $x$ 800).

\section{Discussion:}

The complement system has long been recognized as having a role in immune glomerular disease $8,9,10$. Following the increasing knowledge about the role of complement in the pathophysiology of various diseases, numerous options for therapeutic manipulation of the complement system have been proposed ${ }^{4}$. Therapeutic complement inhibition may be approached at various levels of the complement cascade. An intervention at the level of $\mathrm{C} 3$ inhibits the entire complement system with the possibility of high efficacy but the drawback of an increased risk of infections ${ }^{1}$. 
Complement activation increases tubulointerstitial injury. Complement proteins may access the tubular compartment via glomerular filtration or may be synthesized locally by native renal cells $5,11,12$. Locally synthesized C3 could influence disease progression in several ways. There may be a concentration effect. The alternative pathway is important in this disease model, and its activation is critically dependent on the concentration of complement proteins available. Local C3 synthesis may generate a concentration of $\mathrm{C} 3$ permissive for triggering of this pathway ${ }^{13}$. Another possibility is that local $\mathrm{C} 3$ is produced from the basolateral side of cells into a site that is inaccessible to filtered proteins ${ }^{14}$. Therefore, only locally synthesized C3 will be activated at the basement membrane and within the interstitium ${ }^{9}$. The present study showed the similar findings as shown by Larsen and Brun (1979) ${ }^{11}$, Rajaraman et al. (1984) ${ }^{11}$, Nabir Uddin $(1996)^{3}$, Tabassum et al. $(1997)^{6}$, Pasquariello et al. (2000) ${ }^{14}$, Hossain $(2000)^{13}$ and Das et al. (2008) $)^{7}$.

\section{Conclusion:}

The complement system contributes to renal damage in many of the disease entities encountered by the nephrologist ${ }^{12}$. Sound understanding of the complement system will aid the nephrologist in understanding the pathophysiology of renal disease and provide support in making the correct diagnosis. The ultimate goal of understanding the pathophysiology of glomerular injury is to apply such understanding to the development of therapy ${ }^{8}$. Monitoring complement may offer guidance in therapeutic decisions if interpreted with prudence in the clinical context. Whether therapeutic interventions in the complement system will result in meaningful improvements for our patients still remains to be established.

\section{Acknowledgement:}

We would like to pay our heartfelt gratitude to the authority of Armed Forces Institute of Pathology (AFIP), Dhaka Cantonment, Dhaka, for providing the staining facilities and the fluorescent microscope for studying the histopathology of the renal biopsies.

\section{References:}

1. Berger SP, Roos A, Daha MR. Complement and the kidney. Nephrology Dialysis Transplantation 2005; 20: 2613-9.

2. Sheerin NS, Risley $\mathrm{P}$, Abe K, Tang $Z$, Wong W, Lin $\mathrm{T}$, et al. Synthesis of complement protein C3 in the kidney is an important mediator of local tissue injury. FASEB J 2008; 22: 1065-72.

3. Nabir Uddin M. Pattern of glomerulonephritis in Bangladesh: correlation of light microscopy and immunofluorescent study [Dissertation]. Dhaka: BCPS; 1996.

4. Quigg RJ. Use of complement inhibitors in tissue injury. Trends Mol Med 2002; 8(9): 430-6.

5. McCluskey RT. The value of immunofluorescence in the study of human renal disease. J Exp Med 1971; 134: 242S-55S.

6. Tabassum S, Rahman KM, Mamun KZ, Ahmed ANN, Islam KMN, Rahman H. Immunofluorescent microscopic findings in glomerulonephritis. Bangladesh Med Res Counc Bull 1997; 23(3): 7781.

7. Das RK, Saleh AF, Kabir AN, Talukder SI, Kamal M. Immunofluorescence studies of renal biopsies. Dinajpur Med Coll J 2008; 1(1): 8-13.

8. Welch TR. The complement system in renal diseases. Nephron 2001; 88: 199-204.

9. Nomura A, Morita Y, Maruyama S, Hotta N, Nadai M, Wang L, et al. Role of complement in acute tubulointerstitial injury of rats with aminonucleoside nephrosis. Am J Pathol 1997; 151: 539-47.

10. Turnberg D, Lewis M, Moss J, Xu Y, Botto M, Cook HT. Complement activation contributes to both glomerular and tubulointerstitial damage in adriamycin nephropathy in mice. J Immunol 2006; 177: 4094-4102.

11. Rajaraman S, Pinto JA, Cavallo T. Glomerulonepritis with co-existent immune deposits and anti-basement membrane activity. J Clin Pathol 1984; 37: 176-81.

12. Larsen S, Brun C. Immune deposits in human glomerulopathy. Acta Path Microbial Scand (Sect. A) $1979 ; 87: 321-33$.

13. Hossain T. Immune deposits in glomerular diseases and their clinical, histopathological and immunopathological correlation. [Thesis] IPGMR, University of Dhaka; 2000.

14. Pasquariello A, Innocenti M, Batini V, Rindi S, Moriconi L. Routine immunofluorescence and light microscopy processing with a single renal biopsy specimen: 18 year experience in a single centre. Nephrology 2000; 13: 115-8. 